\title{
Special issue on optimization in disaster relief
}

\author{
Karl F. Doerner • Walter J. Gutjahr • \\ Luk Van Wassenhove
}

(C) The Author(s) 2011. This article is published with open access at Springerlink.com

It has been a real pleasure to edit this special issue. It is quite timely given that this area has gained substantial momentum lately. Part of this is due to some recent and very spectacular large disasters. However, another explanation is that researchers realize that the world has become much more interdependent and subject to risk and therefore studying disasters also brings new insights into better understanding regular supply chain management subject to increased volatility.

We received a large number of applications from which we selected the 15 papers in this issue. We are pleased to note that many submissions were related to real applications or at least showed a real concern for applicability of the results. Unfortunately, we had to turn down a number of good submissions. Some because they did not have a clear enough link with the subject and hence we were not sure about the applicability or relevance of the work to humanitarian operations. A few papers that did not make it to this issue will be published in later regular issues of the journal.

The selection of papers in this issue is very rich and showcases the large variety of challenging research questions related to disaster management. Topics range from forecasting of the impact of disasters and the resulting needs for support, to reliability and repair of disaster supply chains, prepositioning of relief items, inventory and other scarce resource allocation, evacuation of victims, pandemics modeling and more general frameworks for system design. The richness and variety of topics

K. F. Doerner $(\varangle)$

Johannes Kepler University Linz, Linz, Austria

e-mail: karl.doerner@jku.at

W. J. Gutjahr

University of Vienna, Vienna, Austria

L. Van Wassenhove

INSEAD, Fontainebleau, France 
and methodologies to tackle the problems makes this issue a nice introduction for researchers and practitioners interested in learning more about this exciting new field of research.

We hope that you will enjoy reading this special issue as much as we enjoyed putting it together.

The editors.

Karl F. Doerner, Johannes Kepler University Linz

Walter J. Gutjahr, University of Vienna

Luk Van Wassenhove, INSEAD

\section{Forecasting of needs assessment}

The contribution by Rodríguez et al. aims at helping decision makers to quickly assess consequences of a disaster, based on (currently available) uncertain and imprecise data. Appropriate severity assessment in the early stage of a disaster is a crucial factor for an efficient relief operation. For predicting disaster consequences, the authors apply a fuzzy rule-based learning procedure extracting information from a historical database. The obtained fuzzy rules are interpretable by human decision makers, and the method does not impose excessive data requirements, which makes it flexible in application. The focus of the present article is on experimental comparisons of the proposed approach with alternative techniques from statistics and machine learning, such as linear discriminant analysis, classification trees, or support vector machines.

\section{Prepositioning}

Rawls and Turnquist tackle the problem of prepositioning emergency supply with uncertainty about where a natural disaster will occur. The first-stage decisions concern the locations and the capacities of storage centers as well as the allocation of (multicommodity) inventories. The second-stage decisions (to be made after the onset of a disaster) refer to the flow of supplies through the network. Compared to previous work, the authors give more realism to the model by adding service-quality constraints restricting the average shipment distance and ensuring that the probability of covering the overall demand reaches a given minimum level. This results in a chanceconstrained, two-stage mixed integer stochastic programming model. An illustrative case study addresses hurricane risks in the South-East of the USA.

\section{Network reliability and repair}

In the contribution by Günneç and Salman, the assessment of reliability and expected performance of an infrastructure network with respect to disaster events as earthquakes or floods is addressed. Whereas the computation of two-terminal or all-terminal reliability on the assumption of independent link failures is standard in reliability theory (although computationally challenging), much less work has been done to deal with the more realistic case where link failures are dependent. The authors take account of 
spatial failure correlations by a link failure model based on a decomposition of the area under consideration into zones; within each zone, the failure of a link is assumed to imply the failure of weaker links. An efficient algorithm for reliability computation based on this model is provided. The procedure is illustrated by a study of the Istanbul highway system under earthquake risk.

Maya and Sörensen consider the problem of allocating scarce resources to repair a rural road network after it has been damaged by a natural or man-made disaster. They propose a solution approach based on the GRASP and variable neighborhood search that aims to maximize the accessibility of as many people as possible to the main cities or regional centers. The efficiency of their approach is demonstrated by applying it to a set of small and medium size instances and to a large real-life motivated instance.

Nolz et al. consider a problem arising in a post-natural-disaster situation. When the infrastructure in an affected region has been partly destroyed, local inhabitants need to be supplied with relief items. In this paper, the authors focus on the aspect of risk on delivery tours for relief supplies, meaning the threat that potential tours become infeasible because of aftershocks following an earthquake or rising water level after inundations. In order to cope with the specifics of different natural disasters several risk measures are presented. The problem is solved using a metaheuristic based on genetic algorithms and variable neighborhood search. The algorithm is tested on real-world data from the province Manabi in Ecuador.

\section{Evacuation}

The contribution by Kimms and Maassen deals with the evacuation of people from an urban area in the case of a disaster or threat. In order to enable a fast and safe evacuation, traffic flows can be re-directed, and departure times can be decided upon. Kimms and Maassen propose a two-phase approach. In the first phase, traffic routes are optimized by means of an extension of a cell transmission model. In the second phase, based on the network design found in the first phase, a refined simulation is carried out in order to provide the decision maker with a realistic prediction of the evacuation dynamics. The approach is applied to a real-world instance referring to an area near Duisburg, Germany.

In the paper of Huibregtse et al, an optimization method for instructions to evacuate the population of a region by car threatened by a hazard is presented. By giving the optimized instructions to the evacuees, traffic conditions and therefore the evacuation efficiency are optimized. The instructions, containing a departure time, a safe destination, and a route, are created using an optimization method based on ant colony optimization. The main contribution of the proposed optimization method compared to other optimization methods is that it simultaneously optimizes departure time, destination, and route instructions. The effectiveness of the optimized instructions is more than doubled compared to the effectiveness of instructions set up by straightforward rules.

Bish analyzed the planning for a bus-based regional evacuation in case of hurricanes. The paper introduces a model specifically designed for bus-based evacuation planning and two mathematical programming formulations. A heuristic is developed to solve the problem. 
Özdamar describes an efficient planning system for coordinating helicopter operations in disaster relief. This system can be used as a simulation tool in contingency planning for better disaster preparedness and helps to generate plans with estimated data. The proposed system consists of a mathematical model and a route management procedure that post processes the outputs of the model. The system is concerned with helicopter operations that involve last mile distribution and pickups for post disaster medical care and injured evacuation. The proposed modeling system accommodates the special aviation constraints of helicopters and it can handle large-scale helicopter missions. The goal of the system is to minimize the total mission time required to complete the transportation task. A scenario that is based on the post-earthquake damage data provided by the disaster coordination center of Istanbul is used for testing the method.

\section{Inventory and resource allocation}

McCoy and Brandeau develop an inventory model for the supply of a refugee camp or relief operation from a central stockpile. They consider two types of decisions: First, how to optimally partition a given budget between stockpiling and shipping costs, and secondly, how to optimize the quantities of items shipped from the stockpile to the relief operation over time. Uncertainty on actual demands in single time periods is represented by a stochastic model. The objective consists in the minimization of the total expected response penalty over a fixed time horizon, where the penalty function can be of linear or of nonlinear form. This gives a nested optimization problem, for which, based on dynamic programming formulations, some analytical results as well as a heuristic solution technique are provided.

Cotta uses optimization techniques to assign the usage of resources after a mass casualty disaster takes place. Using triage methods, casualties have to be prioritized to receive health care in a limited resource scenario. Aiming to do the greatest good to the greatest number of people, it has to be determined how to make the best use of these resources. They use the pilot method and hyperheuristics for solving this problem. An exhaustive empirical evaluation was performed to analyze the influence on the performance of different performance factors.

Rottkemper et al. developed an optimization model for the supply chain of humanitarian relief items. Here, a sudden change in demand or supply can occur. When overlapping disasters occur, goods must be relocated to existing depots in a way which enables rapid supply to regions with new and urgent demand. At the same time, ongoing operations have to continue, i.e. the other regions should not suffer from shortages, and possible future emergencies. A rolling horizon approach for solving the model is presented, and it is shown that taking into account the possibility of future disruptions can help to balance inventories and to reduce total non-served demand.

\section{Pandemics}

Uribe-Sánchez at al. devote their attention to a special disaster type, namely pandemic influenza. Pharmaceutical mitigation strategies (vaccines and antivirals) as well as 
non-pharmaceutical strategies (social distancing) are considered as means to reduce the spread of the disease. The authors present a simulation-based dynamic optimization model and solve it by a simulation-optimization algorithm. One of the novel features of the proposed model is that the possibility of re-allocation of resources during the evolution of an epidemic is taken into account. Historic pandemic data is used to calibrate the model for an example application to a disease outbreak in Florida. Both a scenario of low and of high disease transmissibility is investigated.

\section{Other}

Trautsamwieser et al. proposed a model for the planning of home health care services in times of natural disasters. For solving real-world sized problems, they developed a variable neighborhood search-based heuristic. Numerical studies with real-life data from three districts in Upper Austria are presented. The comparison of the heuristic solution with an actual route plan shows extensive improvements.

Charles and Lauras take a complementary approach to most other contributions in this volume. Whereas the focus of the latter articles is usually on optimization models for specific aspects of a disaster management operation and on their computational solution, Charles and Lauras elaborate a generic enterprise modeling framework for the whole humanitarian supply chain. This conceptual framework is intended to provide the decision makers with a "business view" of the entire process and to serve as a first step to formalization; more specific OR/MS methods can then be incorporated at suitable places. Besides giving a practically oriented overview of particularities of a humanitarian relief operation in general, the article also outlines a special application to the emergency response activities of the International Federation of the Red Cross and Red Crescent Societies.

Open Access This article is distributed under the terms of the Creative Commons Attribution Noncommercial License which permits any noncommercial use, distribution, and reproduction in any medium, provided the original author(s) and source are credited. 